\title{
Imaging Predictors of Incident Heart Failure: A Systematic Review and Meta-
}

\section{Analysis}

Short title: Imaging predictors of heart failure.

Georgios Georgiopoulos, $\mathrm{PhD}^{\mathrm{a}, \mathrm{b}^{*}}$, Alberto Aimo, $\mathrm{MD}^{\mathrm{c}, \mathrm{d}^{*}}$, Andrea Barison, $\mathrm{MD}, \mathrm{PhD}^{\mathrm{c}, \mathrm{e}}$, Nikolaos Magkas, $\mathrm{MD}^{\mathrm{f}}$, Michele Emdin, $\mathrm{MD}, \mathrm{PhD}^{\mathrm{c}, \mathrm{e}}$, Pier-Giorgio Masci, $\mathrm{MD}, \mathrm{PhD}^{\mathrm{a}}$

${ }^{a}$ School of Biomedical Engineering and Imaging Sciences, King's College London, UK; ${ }^{b}$ Department of Clinical Therapeutics, National and Kapodistrian University of Athens, Greece; 'Institute of Life Science, Scuola Superiore Sant'Anna, Pisa, Italy; ${ }^{\mathrm{d} C a r d i o l o g y}$ Division, University Hospital of Pisa, Italy; ${ }^{\mathrm{e}}$ Cardiology Department, Fondazione Toscana Gabriele Monasterio, Pisa, Italy; ${ }^{\mathrm{f}} 1 \mathrm{st}$ Department of Cardiology, National and Kapodistrian University of Athens. * These authors equally contributed.

Word count: 3,649 (text)

Conflicts of interest: GG was supported by a research fellowship by the European Association of Cardiovascular Imaging. The other Authors do not report any conflicts of interest.

\section{Address for correspondence:}

Alberto Aimo, MD

Cardiology Division, University Hospital of Pisa, and Institute of Life Sciences, Scuola Superiore Sant'Anna

Piazza Martiri della Libertà 33

Phone +393477084391

Email: albertoaimo@libero.it, a.aimo@santannapisa.it 


\begin{abstract}
Background: Preventing the evolution of subclinical cardiac disease into overt heart failure (HF) is of paramount importance. Imaging techniques, particularly transthoracic echocardiography (TTE), are well suited to identify abnormalities in cardiac structure and function which precede the development of HF.
\end{abstract}

Methods: This meta-analysis provides a comprehensive evaluation of 32 studies from 11 individual cohorts which assessed cardiac indices from TTE (63\%), cardiovascular magnetic resonance (CMR; $34 \%$ ), or cardiac computed tomography (CCT; 16\%). Eligible studies focused on measures of left ventricular (LV) geometry and function and were highly heterogeneous.

Results: Among the variables that could be assessed through a meta-analytic approach, LV systolic dysfunction, defined as LV ejection fraction (LVEF) lower than 50\%, and LV dilation were associated with a 5-fold (hazard ratio [HR] 4.76, 95\% confidence interval [CI] 1.85 to 12.26) and 3fold (HR 3.14, 95\% CI 1.37 to 7.19) increased risk of HF development, respectively. Any degree of diastolic dysfunction conveyed an independent, albeit weaker, association with HF (HR 1.48, 95\% CI 1.11-1.96), while there was only a trend for LV hypertrophy in predicting incident HF (HR 2.85, 95\% CI 0.82-9.85).

Conclusions: $\mathrm{LVEF}<50 \%$, LV dilation and diastolic dysfunction are independent predictors of incident HF among asymptomatic individuals, while LV hypertrophy seems less predictive. These findings may serve as framework for implementing imaging-based screening strategies in patients at risk of $\mathrm{HF}$ and inform future studies testing preventive or therapeutic approaches aiming at thwarting or halting the progression from asymptomatic (pre-clinical) to overt HF.

Word count: 244 (abstract)

Keywords: heart failure; predictors; imaging; meta-analysis 


\section{Introduction}

Despite considerable advances in treatment, heart failure (HF) is still associated with high rates of morbidity and mortality, and its prevalence in the United States is expected to increase by up to $30 \%$ in 2030, with a concomitant 2-fold rise in the cost of HF care (1). Prevention of symptomatic HF is therefore one of the priorities of cardiovascular medicine.

The dynamic nature of HF urged the Cardiology community to introduce a new classification scheme comprising asymptomatic patients with cardiovascular risk factors without (stage A) or with LV structural and/or functional abnormalities (stage B) alongside patients with symptomatic (stage C) or end-stage (stage D) HF (2). Compelling evidence exists that the onset of HF may be delayed or prevented through interventions aiming to modify cardiovascular risk factors or by treating asymptomatic patients with abnormalities in left ventricular (LV) structure and/or function, whose prevalence in the general population is far from being negligible. For example, a study on asymptomatic elderly subjects reported a median prevalence of systolic and diastolic LV dysfunction of $5.5 \%$ (range $3.3-9.2 \%$ ) and $36.0 \%$ (range 15.8-52.8\%), respectively (2). Imaging techniques allow early detection of these abnormalities during the diagnostic work-up of asymptomatic patients at high risk of developing HF, such as those with hypertension or diabetes (3-5). Nevertheless, the identification of the imaging parameters most closely associated with the future development of HF is an essential pre-requisite for implementing preventive strategies. This knowledge is also instrumental for planning studies testing interventions aiming at minimizing the progression from asymptomatic (stage A and B) to overt HF (stage C and D). To date, several studies on asymptomatic individuals have explored the predictive value of a single or few imaging parameters using a specific imaging modality in heterogeneous populations (6-37), but a comprehensive evaluation is lacking.

We sought to review available evidence and provide quantitative estimates on the association between imaging parameters and the occurrence of HF based on population studies including asymptomatic subjects. 


\section{Methods}

\section{Systematic review}

In February 2020, articles on imaging predictors of incident HF were searched on the PubMed and EMBASE databases, as detailed in Supplemental Table 1. Reference lists of relevant original research papers and review articles were also manually searched. Articles in English, original research studies, and articles evaluating asymptomatic subjects were selected in a stepwise process. We did not impose restrictions on imaging modality or age of study population. Studies without a prospective arm or with inadequate data on imaging predictors or ambiguous documentation of HF as an endpoint were excluded. Conversely, studies considering HF only as part of a composite outcome were considered for the purposes of the systematic review. Eligible articles were selected independently by 2 Authors (AA and PGM; Supplemental Figure 1). The main characteristics of selected studies are reported in Table 1.

\section{Meta-analysis}

\section{Data extraction and quality assessment}

Among the imaging predictors evaluated, LV dilation (16,35), systolic dysfunction (LV ejection fraction $[\mathrm{LVEF}]<50 \%)(17,18,25,31,33)$, LV hypertrophy $(13,14,19,23)$, and diastolic dysfunction $(25,31)$ were evaluated in studies from at least 2 different cohorts, and could be further synthesized through a meta-analytical approach. Two authors (AA and GG) independently recorded hazard ratios (HRs) and corresponding 95\% confidence intervals (CIs) for the occurrence of HF as the primary outcome. When the same predictor was evaluated in more studies from a single cohort, the study with the largest cohort was selected. We retained HRs from the most extensively adjusted models to control for potential confounders. The definitions of LV dilation, hypertrophy and diastolic dysfunction are reported in Table 2, while Supplemental Table 2 reports the adjusting variables and Supplemental Table 3 the definitions of HF. We also assessed the quality of eligible studies by 
implementing the validated Newcastle-Ottawa Quality Assessment Scale (NOS) (38), converting NOS results into the following categories (39): a) good quality for studies $\geq 7$ stars; b) fair quality for 5-6 stars; and c) poor quality for $\leq 4$ stars. The quality of all meta-analyzed studies was classified as good (Supplemental Table 4). Our meta-analysis was performed according to the checklist of the Meta-analysis of Observational Studies in Epidemiology (40).

\section{$\underline{\text { Data analysis }}$}

We conducted a meta-analysis of eligible studies to derive pooled estimates separately for the associations between LV dilation, systolic dysfunction, LV hypertrophy and diastolic dysfunction and incident HF (as previously defined). We employed a random effects model with the inverse variance method and used the $\mathrm{I}^{2}$ statistic to quantify heterogeneity among studies. Aiming to provide more robust results in the context of limited available studies, we utilized the Paule and Mandel estimator (41). The mean effect size and CIs of individual studies were illustrated with forest plots. In the absence of an adequate number of studies, we did not apply sub-group and meta-regression analyses; accordingly, we did not further explore the presence of potential publication bias. Statistical analysis was performed with STATA v12.1 (StataCorp, College Station, Texas, USA). Two-tailed p values $<0.05$ were deemed significant.

\section{Results}

\section{Selected studies}

The main characteristics of selected studies $(n=32)$ are summarized in Table 1 . All studies evaluated asymptomatic individuals, and 2 studies specifically excluded patients with HF (7,27). Conversely, 2 studies included also patients with history of HF $(30,31)$. However, these studies were considered in the analysis because of the very small proportions of patients with HF (less than 1\%). Seven studies (23\%) evaluated populations of elderly subjects. The studies evaluated 9 cohorts ranging from up to 6,781 subjects (in the Multi-Ethnic Study of Atherosclerosis [MESA]) (8) to 552 subjects (in the 
Uppsala Longitudinal Study of Adult Men) (33) (Table 1). When considering the largest population from each cohort, the total patient number amounted to 25,693 subjects.

The majority of studies ( $\mathrm{n}=20,63 \%)$ evaluated transthoracic echocardiographic (TTE), 11 (34\%), cardiovascular magnetic resonance (CMR) parameters, and $5(16 \%)$ cardiac computed tomography (CCT) parameters; none of the study considered results from cardiac nuclear imaging.

\section{Left ventricular size and shape}

Two CMR studies $(16,35)$ examined the association between LV dilation at baseline and the risk of developing HF in independent cohorts. Collectively, LV dilation was associated with incident HF, with pooled HR 3.14 (95\% CI 1.37 to $\left.7.19 ; I^{2}=59.9 \%\right)$, (Figure 1A).

The first of these 2 studies were based on the MESA cohort and reported that LV dilation (defined as LV end-diastolic diameter $>52 \mathrm{~mm}$ ) predicted incident HF over a median of 12 years. Subjects with LV dilation and LVEF <50\% had the greatest risk of HF (HR 7.35 [2.36 to 22.85]), and those with LV dilation but $\mathrm{LVEF} \geq 50 \%$ had an intermediate risk (HR 2.22 [1.46 to 3.37]) as compared with patients with a non-dilated LV and preserved LVEF (16). Interestingly, a high proportion of subjects with LV dilation had eccentric remodelling, which is a risk factor for HF, as detailed in the following section. In a CCT study on the same population, LV area (measured in mid-diastole and normalized for body surface area [BSA]) displayed a significant association with incident HF (HR 1.10 per 100 $\left.\mathrm{mm}^{2} / \mathrm{m}^{2}, \mathrm{p}<0.001\right)$ after adjustment for clinical variables and coronary artery calcium $(\mathrm{CAC})$ score (8). Conversely, LV end-diastolic diameter at baseline did not independently predict incident including HF among elderly individuals (34).

Regarding LV shape, a high index of sphericity on CMR imaging was a strong, independent predictor of HF in a model including clinical variables, LV end-diastolic volume index, LV mass index, LVEF, and N-terminal fraction of pro-B-type natriuretic peptide (HR 2.13 [1.30 to 3.49]). Even a low index of sphericity, interpreted as suggestive of a "stiffer ventricle", predicted an increased risk of $\mathrm{HF}$ (HR 1.88 [1.12 to 3.14]) (6). 


\section{Left ventricular systolic function}

Asymptomatic LV systolic dysfunction ( $\mathrm{LVEF}<50 \%$ ) was associated with an increased risk of HF, by pooled assessment of 3 studies $(18,25,31)$ yielding a pooled HR $4.76\left(1.85\right.$ to $\left.12.26 ; \mathrm{I}^{2}=78.5 \%\right)$ (Figure 1B).

In the Framingham cohort, systolic dysfunction was associated with a high likelihood of incident HF (HR 4.7 [2.7 to 8.1]) after adjustment for cardiovascular risk factors in the whole population as well as in individuals without prior MI or valvular disease. The risk also increased consistently from mild dysfunction (LVEF 40-50\%: HR 3.3 [1.7 to 6.6]) to moderate-to-severe dysfunction (LVEF $<40 \%$; HR 7.8 [3.9 to 15.6]) (27). Systolic dysfunction seemed also a stronger predictor of HF than diastolic dysfunction (HR 2.33 [1.43 to 3.78$]$ vs. 1.32 [1.01 to 1.71]). Interestingly, LV systolic dysfunction was associated with incident HF with reduced EF (HFrEF), whereas antecedent diastolic dysfunction predicted incident HF with preserved EF (HFpEF) (25).

In elderly individuals from the Olmsted County population, systolic dysfunction was a stronger predictor of HF than diastolic dysfunction (32). In the MESA cohort, subjects with CMR-derived LVEF $<50 \%$ or $50-55 \%$ had a higher risk of HF than those with LVEF $>55 \%$ in a multivariable model including also LV mass (17). A nonlinear relationship between increasing LVEF and HF risk was observed, and individuals with LVEF $<55 \%$ and those with LVEF $>70 \%$ had an increased risk for HF compared with those with LVEF 55\% (17). The likelihood of HF was particularly high in patients with LVEF <50\% and LV dilation, in keeping with other studies (16).

Several indexes of systolic function other than LVEF have been evaluated. Tissue Doppler-derived peak systolic myocardial velocity by TTE was a strong predictor of HF, and retained independent prognostic significance even when adjusting for LVEF (HR 1.26 [1.03 to 1.54 ] per $1 \mathrm{~cm} / \mathrm{s}$ decrease) (30). Circumferential strain from speckle tracking TTE predicted incident HF in a model including also LV fractional shortening, a metric of systolic function, and LV mass index (HR 1.79 [1.35 to 2.37] per standard deviation increment), while the other measures from speckle tracking analysis were not independently associated with HF onset (24). In a tagged CMR study, both the average 
circumferential strain from 12 segments (anterior, lateral, posterior and septal, at the basal, mid-cavity and apical levels), and the average circumferential strain from mid-cavity segments displayed an independent prognostic value for incident HF, regardless of clinical variables, LV mass index and LVEF, and even in subjects with LVEF $\geq 50 \%$ (11). Finally, LV ejection time (i.e., the duration of the systolic flow through the LV outflow tract by pulsed-wave Doppler) (29) and myocardial performance index (a measure providing a comprehensive assessment of systolic and diastolic function) $(28,33)$ were reported to predict incident HF.

\section{Left ventricular hypertrophy and mass}

We performed a meta-analytic assessment of 2 studies considering LV hypertrophy as predictor of HF $(13,19)$. While both studies showed an independent prognostic value of LV hypertrophy for HF onset, the pooled estimate failed to confirm the results of the original studies (pooled HR 2.85 as compared to subjects without LV hypertrophy [0.82 to 9.85], $\mathrm{p}=0.125$ ) (Figure 2A). Significant heterogeneity was observed in the pooled analysis $\left(\mathrm{I}^{2}=97.4 \%\right)$.

In the MESA cohort, LV hypertrophy at CMR (defined as LV mass percentage of predicted greater than the $95^{\text {th }}$ percentile) was associated with incident HF after adjustment for clinical variables and the CAC score (HR 5.4 [3.8 to 7.5]) (13), and the risk of HF increased in parallel with LV mass (9). Additionally, LV mass index refined HF prediction over LVEF alone, and seemed particularly informative in patients with LVEF $<50 \%$ (18). In another cohort, LV hypertrophy (defined on TTE using different cut-offs) emerged as an independent predictor of HF $(19,23)$, with a likelihood of incident HF increasing by $1 \%$ per each $1 \%$ increase in excess of LV mass (21). LV mass (22), LV mass/height (32) and LV mass/BSA (34) were reported to predict first cardiovascular events, including HF.

Among patients with LV hypertrophy (defined as increased LV mass/height), different patterns of remodelling predicted HF development after adjustment for clinical variables: a "dilated" pattern (increased LVEDV/BSA without increased concentricity: HR 5.5 [2.0 to 14.9]), a "thick" pattern 
(increased concentricity without increased LVEDV/BSA: HR 2.2 [1.3 to 3.7], and a "both thick and dilated" pattern (increased concentricity and LVEDV/BSA: HR 4.6 [1.4 to 15.7]) (35). Very limited information is available on the changes in LV geometry and function over time. Most notably, the development of an abnormal LV geometric pattern (relative wall thickness ratio $\geq 80^{\text {th }}$ percentile) over 4 years was associated with an increased risk of cardiovascular events (HF, myocardial infarction, and death) during a 12-year follow-up), and subjects with persistent concentric or eccentric hypertrophy had the highest risk (26).

\section{Diastolic dysfunction}

Two studies evaluating the presence of diastolic dysfunction could be meta-analysed $(25,31)$; their definition of diastolic dysfunction is reported in Supplemental Table 2. Diastolic dysfunction emerged as an independent predictor of incident HF: pooled HR $1.48\left(1.11\right.$ to $\left.1.96 ; \mathrm{I}^{2}=0.0 \%\right)$ (Figure 2B).

In the Framingham cohort, diastolic dysfunction was associated with an increased risk of incident HF regardless of cardiovascular risk factors and systolic dysfunction (HR 1.32 [1.01 to 1.71]) (25). Altered diastolic filling by TTE (defined as higher peak E and higher or lower E/A ratios at mitral valve pulse-wave Doppler sampling) displayed an independent prognostic value for HF onset (20). Furthermore, the E/e' ratio predicted $\mathrm{HF}$ independent of gender and age, as well as of further variables including prior admission for HF and plasma N-terminal fraction of pro-B-type natriuretic peptide (HR 1.66 [1.16 to 2.36]), while the independent prognostic value was lost including LVEF (30). Importantly, the changes in diastolic function over time have been reported to hold prognostic significance. Among 1402 subjects undergoing two TTE examinations with a mean interval of 4 years, and then followed-up for 6 years, HF occurred in $2.6 \%, 7.8 \%$, and $12.2 \%$ of participants in whom diastolic function normalized or remained normal, remained or progressed to mild dysfunction, or remained or progressed to moderate or severe dysfunction, respectively $(p<0.001)(31)$. 


\section{Findings from cardiac computed tomography}

Non-contrast enhanced CCT was evaluated only in the MESA cohort. Baseline CAC score predicted incident HF after adjustment for cardiovascular risk factors or imaging-derived measures, although appeared a much weaker predictor of incident HF than LV mass (12). An increase in CAC by 10 Agatston units per year was associated with a $2 \%$ higher risk of HF over 10 years independent of clinical variables and baseline CAC, even when patients with coronary artery disease were excluded (7). Pericardial fat volume predicted incident HF regardless of cardiovascular risk factors (HR 1.27 [1.12 to 1.45]), while hepatic fat volume did not have independent prognostic significance for this endpoint (15).

\section{Discussion}

To our knowledge, this is the first systematic review and meta-analysis to search for imaging predictors of incident HF in subjects from the general population, i.e. beyond specific disease settings such as systemic hypertension, primary mitral regurgitation, aortic stenosis, or hypertrophic cardiomyopathy. Among the variables that could be assessed through a meta-analytic approach, LV systolic dysfunction (LVEF < 50\%) and dilatation (increased LV diastolic dimension, defined through different criteria) displayed a strong independent prognostic value resulting in about 5- and 3-fold increased likelihood of incident HF, respectively. Diastolic dysfunction by TTE had an independent, although less strong, association with HF development, and LV hypertrophy seemed to be another weak predictor, not achieving statistical significance in the pooled analysis (Central Illustration).

A systematic search of studies assessing imaging predictors of incident HF allowed to identify 30 studies, 7 of them considering only elderly subjects (Table 1). The majority of retrieved studies (60\%) analysed TTE parameters, in keeping with the role of TTE as the first-line examination in patients with suspected cardiac disease. A substantially lower number of studies considered findings from CMR or CCT, reflecting the still limited availability and relatively high costs of these techniques with 
respect to TTE. Several imaging modalities were investigated in the MESA cohort (6-18), although no head-to-head comparison between the different techniques has been carried out so far.

Overall, our meta-analysis confirmed the findings of individual studies. First, systolic function, evaluated through absolute LVEF or cut-off values (most commonly 50\%), is related with incidental HF independently from LV dimension and mass. Notably, LVEF $<50 \%$ seems to be a particularly strong predictor of $\mathrm{HF}$, exceeding the predictive significance of LV dilatation or hypertrophy. Furthermore, based on an individual study, systolic dysfunction independently predicted the evolution towards HFrEF, while diastolic dysfunction was more predictive of incident HFpEF (25).

LV dilation is a compensatory mechanism for increased diastolic wall stress, and leads to exhaustion of the Frank-Starling mechanism for acute compensation of flow. LV hypertrophy develops as a response to increased systolic wall stress and usually culminate in impaired output. Systolic dysfunction then testifies an exhaustion of compensatory mechanisms, which may explain why an impaired LVEF is a stronger predictor of HF as compared to an increased LV end-diastolic volume or mass. Stand-alone diastolic dysfunction is rarely present on the long term, and it is usually accompanied by pump dysfunction. Diastolic dysfunction is a weaker predictor of HF as compared to depressed LVEF. This may be ascribed to two main factors: 1) the difficulty to obtain accurate and precise estimate of the diastolic function by current imaging techniques (42), 2) the heterogeneous diagnostic criteria across the studies (Supplemental Table 2) (43). Similar considerations may explain the lower prognostic value of LV hypertrophy in pooled analysis, which was measured at CMR in one study (15) and calculated using Devereux formula using TTE in second one (17)

Only a few studies have evaluated the prognostic value of abnormal LV kinesis at speckle-tracking TTE or CMR strain analysis in asymptomatic subjects $(11,24)$. Similarly, limited information is available for CAC score, its progression and incident HF. Either CAC score or its evolution are weak predictors of HF despite a good performance for the prediction of coronary artery disease $(7,12)$. These findings may be explained by the absence of a direct pathophysiological link between the burden of coronary artery calcium and HF development. Additionally, the prognostic value of CAC 
score may be diluted by the adjustment for cardiovascular risk factors such as diabetes (12), which are strongly associated with higher CAC score values (42), and are important risk factors of HF development (5).

Our findings provide a conceptual framework for an imaging-based approach for prevention or treatment of patients at the early pre-symptomatic stages of HF (stage A and B of HF). Given its high availability and limited cost, point-of-care TTE may be potentially used as screening tool. A simple assessment of LVEF and of LV end-diastolic diameter or volume would identify patients with structural heart disease (stage $\mathrm{B}$ of $\mathrm{HF}$ ) at risk of incident HF. A more intensive intervention on modifiable cardiovascular risk factors, and the commencement or up-titration of anti-remodelling therapy could substantially reduce the risk of $\mathrm{HF}$, potentially leading to recovery or normalization of cardiac geometry and function (43). Dedicated studies are warranted to explore the feasibility and cost-effectiveness of this strategy. Despite being less available and more expensive, CMR may potentially implement the identification and stratification of subjects in stage A and B of HF given the higher accuracy and reproducibility of this modality for LV structural and functional parameters as compared to 2-dimensional TTE (46). Our meta-analysis could also provide the basis for the creation of a risk score to predict the future development of HF. Notably, changes in score values from baseline could also be regarded as a surrogate endpoint for intervention acting on risk factors for HF, such as hypertension or diabetes. Finally, it has to be acknowledged that further research is needed to investigate the predictive value of more advanced techniques, such as tissue characterization by CMR using quantitative parametric imaging and late gadolinium enhancement, which enable a thorough characterization of myocardial tissue composition (47).

Some limitations should be acknowledged in our study. First, a high degree of heterogeneity was observed among studies with respect to parameters evaluated, multivariate models, and outcomes (often not including incident HF alone); this greatly reduced the feasibility of a meta-analytic assessment. Even the most commonly explored parameters were not evaluated in a consistent way across studies; for example, the increase in LV mass was variably considered as absolute or indexed 
values (21,22,32), and arbitrary cut-offs for LV hypertrophy $(19,23)$, or specific patterns of hypertrophy have been considered (35). Most notably, the fact that 2 positive studies regarding LV hypertrophy did not pool to a global positive result, in an analysis with a high degree of heterogeneity, should not directly be interpreted as failure to predict incident HF. However, it should be noted that a small number of studies may result in underestimation of between-study variance and falsely narrow CIs might be derived (48) if a robust estimator of heterogeneity is not implemented. In view of its superiority for dichotomous outcomes, we employed the Paule and Mandel estimator instead of the popular DerSimonian and Laird (48) and calculated wider CIs for the main predictors of the metaanalysis. Second, parameters such as left atrial (LA) volume index or variables from tissue Doppler imaging might hold prognostic significance, and novel measures of cardiac function such as LV global longitudinal strain and LA strain are promising predictors of incident cardiovascular disease $(44,45)$. Adding this information might allow to refine the prediction of new-onset HF based on measures of cardiac structure and function. Unfortunately, data currently available in the general population do not allow to perform a meta-analytic assessment of their predictive performance. Third, residual confounding from uncontrolled parameters in the multivariable models cannot be excluded. Finally, the occurrence of "incident HF" was either adjudicated by study investigators or established based on administrative data (Supplemental Table 4), and the studies did not distinguish between HF with reduced or mid-range/preserved EF, although imaging predictors of these 2 conditions might differ.

In conclusion, contemporary studies on predictors of HF have focused mostly on measures of LV geometry and function. Their cumulative assessment reveals that asymptomatic abnormalities, particularly depressed LVEF ( $<50 \%)$, LV dilation and diastolic dysfunction, and to a lesser extent LV hypertrophy, predict HF development. These findings could inform future studies on imagingbased markers aiming at implementing preventive or treatment strategies in patients at early presymptomatic stages of HF. 


\section{Figure legend}

Figure 1. Left ventricular (LV) dilation (A) and systolic dysfunction (B) as predictors of heart failure in asymptomatic individuals.

Hazard ratio (HR) and 95\% confidence intervals (CIs) for LV dilation and LV ejection fraction (LVEF) $<50 \%$ are reported. Studies are listed by date of publication. Boxes represent the HR and lines represent the $95 \%$ CIs for individual studies. The diamonds and their width represent the pooled HRs and the $95 \%$ CIs, respectively. Pooled estimates are derived from a random-effects model with the Hartung-Knapp correction to account for the small number of studies. The definitions of LV dilation and the variables considered for adjustment are reported in Supplemental Table 2.

Figure 2. Left ventricular (LV) hypertrophy (A) and diastolic dysfunction (B) as predictors of heart failure in asymptomatic individuals.

Hazard ratio (HR) and 95\% confidence intervals (CIs) for LV hypertrophy and diastolic dysfunction (any degree) are reported. Studies are listed by date of publication. Boxes represent the HR and lines represent the $95 \%$ CIs for individual studies. The diamonds and their width represent the pooled HRs and the 95\% CIs, respectively. Pooled estimates are derived from a random-effects model with the Hartung-Knapp correction to account for the small number of studies. The definitions of LV hypertrophy and the variables considered for adjustment are reported in Supplemental Table 2.

Central Illustration. Imaging predictors of incident heart failure (HF).

Left ventricular (LV) hypertrophy is reported in Italic since it did not emerge as an independent predictor of incident $\mathrm{HF}$ in the dedicated meta-analysis. Hazard ratio (HR) values and the corresponding 95\% confidence intervals are derived from the meta-analytical assessment (Figures 2 and 3). 


\section{References}

1. Heidenreich PA, Albert NM, Allen LA et al. Forecasting the impact of heart failure in the United States: a policy statement from the American Heart Association. Circ Heart Fail 2013; 6:606-619.

2. van Riet EE, Hoes AW, Wagenaar KP, Limburg A, Landman MA, Rutten FH. Epidemiology of heart failure: the prevalence of heart failure and ventricular dysfunction in older adults over time. A systematic review. Eur J Heart Fail 2016; 18:242-252.

3. Kosmala W, Marwick TH. Asymptomatic left ventricular diastolic dysfunction: predicting progression to symptomatic heart failure. JACC Cardiovasc Imaging 2020; 13: 215-227.

4. Williams B, Mancia G, Spiering W et al. 2018 ESC/ESH Guidelines for the management of arterial hypertension. Eur Heart J 2018; 39: 3021-3104.

5. Cosentino F, Grant PJ, Aboyans V et al. 2019 ESC Guidelines on diabetes, pre-diabetes, and cardiovascular diseases developed in collaboration with the EASD. Eur Heart J 2020; 41: 255-323.

6. Ambale-Venkatesh B, Yoneyama K, Sharma RK et al. Left ventricular shape predicts different types of cardiovascular events in the general population. Heart 2017; 103: 499-507.

7. Bakhshi H, Ambale-Venkatesh B, Yang X et al. Progression of Coronary Artery Calcium and Incident Heart Failure: The Multi-Ethnic Study of Atherosclerosis. JAHA 2017; 6.

8. Bittencourt MS, Blankstein R, Mao S et al. Left ventricular area on non-contrast cardiac computed tomography as a predictor of incident heart failure - The Multi-Ethnic Study of Atherosclerosis. J Cardiovasc Comput Tomogr 2016; 10: 500-506.

9. Bluemke DA, Kronmal RA, Lima JA et al. The relationship of left ventricular mass and geometry to incident cardiovascular events: the MESA (Multi-Ethnic Study of Atherosclerosis) study. J Am Coll Cardiol 2008; 52: 2148-2155. 
10. Broughton ST, O'Neal WT, Al-Mallah M et al. Normal findings on noninvasive cardiac assessment and the prediction of heart failure: The Multi-Ethnic Study of Atherosclerosis (MESA). Int J Cardiol 2017; 249: 308-312.

11. Choi EY, Rosen BD, Fernandes VR et al. Prognostic value of myocardial circumferential strain for incident heart failure and cardiovascular events in asymptomatic individuals: the Multi-Ethnic Study of Atherosclerosis. Eur Heart J 2013; 34: 2354-2361.

12. Jain A, McClelland RL, Polak JF et al. Cardiovascular imaging for assessing cardiovascular risk in asymptomatic men versus women: the multi-ethnic study of atherosclerosis (MESA). Circ Cardiovasc Imaging 2011; 4: 8-15.

13. Kawel-Boehm N, Kronmal R, Eng J et al. Left ventricular mass at MRI and long-term risk of cardiovascular events: the Multi-Ethnic Study of Atherosclerosis (MESA). Radiology 2019; 293: $107-114$

14. Oseni AO, Qureshi WT, Almahmoud MF et al. Left ventricular hypertrophy by ECG versus cardiac MRI as a predictor for heart failure. Heart 2017; 103: 49-54.

15. Shah RV, Anderson A, Ding J et al. Pericardial, but not hepatic, fat by CT is associated with CV outcomes and structure: the Multi-Ethnic Study of Atherosclerosis. JACC Cardiovasc Imaging 2017; 10: 1016-1027.

16. Yeboah J, Bluemke DA, Hundley WG, Rodriguez CJ, Lima JA, Herrington DM. Left ventricular dilation and incident congestive heart failure in asymptomatic adults without cardiovascular disease: multi-ethnic study of atherosclerosis (MESA). J Card Fail 2014; 20: 905-911.

17. Yeboah J, Rodriguez CJ, Qureshi W et al. Prognosis of low normal left ventricular ejection fraction in an asymptomatic population-based adult cohort: the Multiethnic Study of Atherosclerosis. J Card Fail 2016; 22: 763-768. 
18. Yeboah J, Rodriguez CJ, Stacey B et al. Prognosis of individuals with asymptomatic left ventricular systolic dysfunction in the multi-ethnic study of atherosclerosis (MESA). Circulation 2012; 126: 2713-2719.

19. Almahmoud MF, O'Neal WT, Qureshi W, Soliman EZ. Electrocardiographic versus echocardiographic left ventricular hypertrophy in prediction of congestive heart failure in the elderly. Clin Cardiol 2015; 38: 365-370.

20. Aurigemma GP, Gottdiener JS, Shemanski L, Gardin J, Kitzman D. Predictive value of systolic and diastolic function for incident congestive heart failure in the elderly: the cardiovascular health study. J Am Coll Cardiol 2001; 37: 1042-1048.

21. de Simone G, Gottdiener JS, Chinali M, Maurer MS. Left ventricular mass predicts heart failure not related to previous myocardial infarction: the Cardiovascular Health Study. Eur Heart J 2008; 29: 741-747.

22. Hoang K, Zhao Y, Gardin JM et al. LV mass as a predictor of CVD events in older adults with and without metabolic syndrome and diabetes. JACC Cardiovasc Imaging 2015; 8: $1007-1015$

23. Zile MR, Gaasch WH, Patel K, Aban IB, Ahmed A. Adverse left ventricular remodeling in community-dwelling older adults predicts incident heart failure and mortality. JACC Heart Fail 2014; 2: 512-22.

24. Cheng S, McCabe EL, Larson MG et al. Distinct aspects of left ventricular mechanical function are differentially associated with cardiovascular outcomes and all-cause mortality in the community. JAHA 2015; 4: e002071.

25. Lam CS, Lyass A, Kraigher-Krainer E et al. Cardiac dysfunction and noncardiac dysfunction as precursors of heart failure with reduced and preserved ejection fraction in the community. Circulation 2011; 124: 24-30. 
26. Lieb W, Gona P, Larson MG et al. The natural history of left ventricular geometry in the community: clinical correlates and prognostic significance of change in LV geometric pattern. JACC Cardiovasc Imaging 2014; 7: 870-878.

27. Wang TJ, Evans JC, Benjamin EJ, Levy D, LeRoy EC, Vasan RS. Natural history of asymptomatic left ventricular systolic dysfunction in the community. Circulation 2003; 108: 977-982.

28. Biering-Sorensen T, Mogelvang R, Jensen JS. Prognostic value of cardiac time intervals measured by tissue Doppler imaging M-mode in the general population. Heart 2015; 101: 954-60.

29. Biering-Sorensen T, Querejeta Roca G, Hegde SM et al. Left ventricular ejection time is an independent predictor of incident heart failure in a community-based cohort. Eur J Heart Fail 2018; 20: 1106-1114.

30. Mogelvang R, Biering-Sorensen T, Jensen JS. Tissue Doppler echocardiography predicts acute myocardial infarction, heart failure, and cardiovascular death in the general population. Eur Heart J Cardiovasc Imaging 2015; 16: 1331-1337.

31. Kane GC, Karon BL, Mahoney DW et al. Progression of left ventricular diastolic dysfunction and risk of heart failure. JAMA 2011; 306: 856-863.

32. Tsang TS, Barnes ME, Gersh BJ et al. Prediction of risk for first age-related cardiovascular events in an elderly population: the incremental value of echocardiography. J Am Coll Cardiol 2003; 42: 1199-1205.

33. Arnlov J, Ingelsson E, Riserus U, Andren B, Lind L. Myocardial performance index, a Doppler-derived index of global left ventricular function, predicts congestive heart failure in elderly men. Eur Heart J 2004; 25: 2220-2225.

34. Lind L, Sundstrom J. Change in left ventricular geometry over 10 years in the elderly and risk of incident cardiovascular disease. J Hypert 2019; 37: 325-330. 
35. Garg S, de Lemos JA, Ayers C et al. Association of a 4-tiered classification of LV hypertrophy with adverse cv outcomes in the general population. JACC Cardiovasc Imaging 2015; 8: 1034-1041.

36. Gori M, Lam CS, D'Elia E et al. Integrating natriuretic peptides and diastolic dysfunction to predict adverse events in high-risk asymptomatic subjects. Eur J Prev Cardiol 2020: 2047487319899618.

37. Shah AM, Claggett B, Kitzman D et al. Contemporary assessment of left ventricular diastolic function in older adults: the Atherosclerosis Risk in Communities Study. Circulation 2017; 135: 426-439.

38. Stang A. Critical evaluation of the Newcastle-Ottawa scale for the assessment of the quality of nonrandomized studies in meta-analyses. Eur J Epidemiol 2010; 25: 603-605.

39. Chiriaco M, Pateras K, Virdis A et al. Association between blood pressure variability, cardiovascular disease and mortality in type 2 diabetes: A systematic review and metaanalysis. Diab Ob Metab 2019; 21: 2587-2598.

40. Stroup DF, Berlin JA, Morton SC et al. Meta-analysis of observational studies in epidemiology: a proposal for reporting. Meta-analysis Of Observational Studies in Epidemiology (MOOSE) group. JAMA 2000; 283: 2008-2012.

41. Partlett C, Riley RD. Random effects meta-analysis: Coverage performance of 95\% confidence and prediction intervals following REML estimation. Stat Med 2017; 36: 301-317.

42. Thomas IC, Shiau B, Denenberg JO et al. Association of cardiovascular disease risk factors with coronary artery calcium volume versus density. Heart 2018; 104: 135-143.

43. Goldberg LR, Jessup M. Stage B heart failure: management of asymptomatic left ventricular systolic dysfunction. Circulation 2006; 113: 2851-2860.

44. Biering-Sørensen T, Biering-Sørensen SR, Olsen FJ et al. Global longitudinal strain by echocardiography predicts long-term risk of cardiovascular morbidity and mortality in a lowrisk general population: the Copenhagen City Heart Study. Circ Cardiovasc Imaging 2017;10. 
45. Modin D, Biering-Sørensen SR, Møgelvang R, Alhakak AS, Jensen JS, Biering-Sørensen T. Prognostic value of left atrial strain in predicting cardiovascular morbidity and mortality in the general population. Eur Heart J Cardiovasc Imaging 2019; 20: 804-815. 